\title{
Rituximab in Combination With Fludarabine and Cyclophosphamide in the Treatment of Patients With Recurrent Follicular Lymphoma
}

\author{
Stefano Sacchi, mo ${ }^{1}$ \\ Samantha Pozzi, MD ${ }^{1}$ \\ Raffaella Marcheselli, ss ${ }^{1}$ \\ Massimo Federico, MD ${ }^{1}$ \\ Alessandra Tucci, $\mathrm{MD}^{2}$ \\ Francesco Merli, $\mathrm{MD}^{3}$ \\ Loretta Orsucci, $\mathrm{MD}^{4}$ \\ Marina Liberati, $\mathrm{MD}^{5}$ \\ Daniele Vallisa, ${ }^{6}{ }^{6}$ \\ Maura Brugiatelli, мо ${ }^{7}$ \\ On behalf of the Italian Lymphoma \\ Study Group (GISL)

\footnotetext{
${ }^{1}$ Department of Oncology and Hematology, University of Modena, Modena, Italy.

${ }^{2}$ Department of Internal Medicine, Brescia Hospital, Brescia, Italy.

${ }^{3}$ Department of Hematology, Santa Maria Nuova Hospital, Reggio Emilia, Italy.

${ }^{4}$ Department of Hematology, Molinette Hospital, Torino, Italy.

${ }^{5}$ Institute of Oncological Sciences, University of Perugia, Perugia, Italy.

${ }^{6}$ Department of Hematology and Oncology, Piacenza Hospital, Piacenza, Italy.

${ }^{7}$ Division of Hematology, Papardo Hospital, Messina, Italy.
}

\footnotetext{
Supported by the Associazione Angela Serra per la Ricerca sul Cancro.

Preliminary and final results presented at the American Society of Hematology Annual Meeting, Philadelphia, Pennsylvania, December 6-10, 2002; San Diego, California, December 6-9, 2003; and Orlando, Florida, December 9-12, 2006.
}

BACKGROUND. The current study was conducted to asses the safety profile and clinical activity of rituximab in combination with fludarabine and cyclophosphamide in patients with recurrent follicular lymphoma (FL).

METHODS. This study was a noncomparative, multicenter, phase II study. Between March 2000 and December 2002, 54 patients with recurrent FL were enrolled in the FC+R trial. Patients received fludarabine at a dose of $25 \mathrm{mg} / \mathrm{m}^{2}$ and cyclophosphamide at a dose of $300 \mathrm{mg} / \mathrm{m}^{2}$ daily for 3 consecutive days, every 3 weeks for 4 cycles. Rituximab was administered at a dose of $375 \mathrm{mg} / \mathrm{m}^{2}$ beginning 2 weeks after the first course of fludarabine and cyclophosphamide and then on Day 1 of each cycle thereafter. The planned treatment duration was 10 weeks.

RESULTS. Overall, $92 \%$ of patients completed the planned therapy in 10 to 14 weeks and $74 \%$ achieved a complete response (CR). Among patients with BCL2positive bone marrow, $86 \%$ obtained a molecular disease remission (MR). The median survival from treatment (SFT), the duration of disease remission (DR), and time to disease progression (TTP) had not been reached after a median follow-up of 45 months. Of the baseline characteristics, $>2$ previous treatments, BCL2-positive bone marrow, and low Follicular Lymphoma International Prognostic Index (FLIPI) score were found to be associated with better DR and/or TTP. Hematologic toxicity was transient and reversible, with the exception of 3 patients with severe and prolonged neutropenia. Three patients presented with infections, 1 of whom died of bronchopneumonia.

CONCLUSIONS. The FC+R scheme, a nonanthracycline-containing regimen lasting up to 10 weeks, was found to be relatively well-tolerated and demonstrated significant antilymphoma activity with excellent clinical CR and molecular response rates. Cancer 2007;110:121-8. ㄷ) 2007 American Cancer Society.

KEYWORDS: non-Hodgkin lymphoma, follicular lymphoma, rituximab, fludarabine, cyclophosphamide, recurrent.

E ollicular non-Hodgkin lymphoma is classified in the group of indolent lymphomas that by definition are considered to be associated with a survival measured in years, independent of whether any therapy is given. ${ }^{1}$ The natural history of these lymphomas is

We thank the GISL trial office staff and Luigi Marcheselli, MS, in particular for statistical analysis of the data and Orsola Ciarcia and Caterina Mammi, BS, for central data management.

Address for reprints: Sacchi Stefano, MD, Centro Oncologico Modenese, Policlinico, Largo del Pozzo
71, 4100 Modena, Italy; Fax: (011) 39059422 3707; E-mail: ssacchi@unimo.it

Received December 27, 2006; revision received February 14, 2007; accepted February 15, 2007. 
characterized by a high initial response rate to chemotherapy followed invariably by disease recurrence, with subsequent remissions of progressively shorter duration. ${ }^{2,3}$ The majority of patients eventually die of their disease. Follicular lymphoma (FL) is often characterized by the $t(14 ; 18)$ translocation, in which the BCL2 protooncogene on chromosome 18 is translocated to the immunoglobulin heavy chain $(\mathrm{IgH})$ region on chromosome 14, creating a hybrid BCL2/ IgH gene. ${ }^{4}$ This translocation results in the overexpression and activation of BCL2 and a subsequent inhibition of apoptosis.

To our knowledge, to date there is no gold standard for the treatment of patients with newly diagnosed or recurrent advanced FL. Until a few years ago, the management of FL was basically palliative and little therapeutic progress appears to have been made within the last 30 years. $^{2}$

A large majority of FL patients initially respond to alkylating agent-based combination chemotherapy. ${ }^{5}$ The use of purine analogues ${ }^{6}$ and combination regimens containing anthracyclines ${ }^{7}$ may increase the rate of complete response (CR). Adding interferon in the context of relatively intensive initial chemotherapy may prolong overall survival, ${ }^{8}$ but many patients are unable to tolerate the accompanying side effects. ${ }^{9}$ The results of a recent study suggested that the combination of cyclophosphamide, doxorubicin, vincristine, and prednisone plus rituximab $(\mathrm{CHOP}+\mathrm{R})$ may prolong the duration of disease remission (DR). ${ }^{10}$ Although the results of recent retrospective studies ${ }^{9,11}$ suggest that new treatment options, including the incorporation of rituximab, have made significant stepwise improvements in patient outcome, to our knowledge it has not been shown conclusively that these treatment modalities improve survival in FL patients.

New treatment approaches for FL currently under investigation include attempts to eradicate the disease using high-dose chemotherapy with autologous stem cell transplantation, and testing new drugs or biologic therapies. Among these, the use of antiCD20 monoclonal antibodies (MoAbs) appears to be the most useful approach. The chimeric anti-CD20 MoAb rituximab has been shown to be a highly effective agent in the treatment of FL, either alone ${ }^{12}$ or in combination with chemotherapy ${ }^{9-11,13}$ or other biologic agents. ${ }^{14}$ The ability of rituximab to sensitize indolent lymphoma-derived cell lines to cytotoxic chemotherapy agents has been demonstrated previously. ${ }^{15,16}$ Furthermore, chemotherapeutic agents such as fludarabine also may sensitize cells to the effects of rituximab. ${ }^{17}$ Cyclophosphamide and fludarabine have shown synergistic activity in B-cell chronic lymphocytic leukemia in vitro. ${ }^{18}$ In view of the single-agent activity and demonstrated synergy between cyclophosphamide and fludarabine, and between fludarabine and rituximab, we evaluated the combination of fludarabine and cyclophosphamide plus rituximab $(\mathrm{FC}+\mathrm{R})$ in previously treated patients with advanced FL. The primary objective of the current study was to assess the safety profile and clinical activity of the $\mathrm{FC}+\mathrm{R}$ combination. The secondary goal was to evaluate the ability of the treatment to convert bone marrow BCL2 positivity such that patients achieved molecular disease remissions (MRs).

\section{MATERIALS AND METHODS Patients and Entry Criteria}

This study was conducted at 10 Gruppo Italiano Studio Linfomi (GISL) sites in Italy between March 2000 and December 2002; a total of 54 patients were enrolled in this trial. Ten patients, 25 patients, and 19 patients were enrolled during 2000, 2001, and 2002, respectively. Eligible patients had histologically documented World Health Organization (WHO) grade 1$2^{1}$, CD20-positive, recurrent FL. Patients were required to have an Ann Arbor stage III or IV disease. Patients with stage II disease were eligible if they presented with B symptoms or bulky disease. Patients were further required to have a need for therapy in the opinion of the treating clinician, measurable disease, an expected survival of $\geq 6$ months, to be aged 18 to 70 years, to have undergone $<3$ lines of chemotherapy, and to have an Eastern Cooperative Oncology Group (ECOG) performance status of 0 to 2 . Patients with known human immunodeficiency virus (HIV) infection; hepatitis B or C virus; central nervous system (CNS) lymphoma; previous malignancies; or cardiac, renal, hepatic, or respiratory failure were excluded. Pregnant or lactating women and patients of childbearing potential were not eligible for the current study, unless birth control measures were used. The study complied with all requirements of the Declaration of Helsinki and its current amendments, and was conducted in accordance with the Good Clinical Practice guidelines. The protocol was approved by the institutional review board at each participating center. Written informed consent was obtained from all patients.

\section{Study Design and Treatment Schedule}

This was an open-label, noncomparative, multicenter, phase II study. The dose and schedule of rituximab in combination with fludarabine and cyclophosphamide $(\mathrm{FC}+\mathrm{R})$ is illustrated in Figure 1. 
Treatment schema
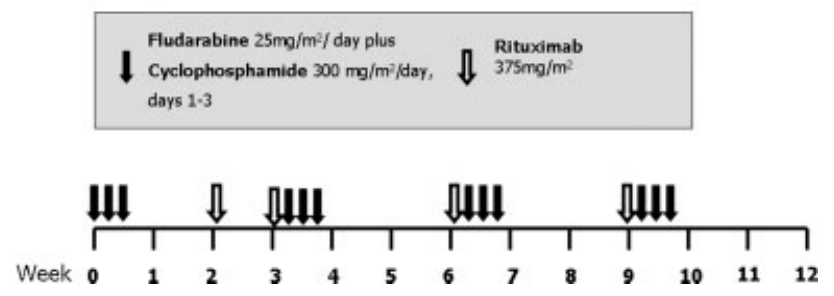

FIGURE 1. Dosing schedule of the regimen of rituximab in combination with fludarabine and cyclophosphamide.

Briefly, patients received $\mathrm{FC}+\mathrm{R}$ chemoimmunotherapy, comprised of fludarabine at a dose of $25 \mathrm{mg} / \mathrm{m}^{2}$ and cyclophosphamide at a dose of $300 \mathrm{mg} / \mathrm{m}^{2}$ daily for 3 consecutive days every 3 weeks for 4 cycles. Rituximab at a dose of $375 \mathrm{mg} / \mathrm{m}^{2}$ was administered by infusion beginning 2 weeks after the first course of fludarabine and cyclophosphamide to reduce the risk of cytokine release syndrome, and was then administered on Day 1 of each cycle thereafter. Treatment was planned for 10 weeks. Oral trimethoprim-sulfamethoxazole (TMP-SMX), acyclovir prophylaxis, and granulocyte-colony-stimulating factor (G-CSF) support were allowed at the discretion of the treating physician. Concurrent treatment with corticosteroids was not permitted.

\section{Patient Evaluation, Follow-up and Response Criteria}

Pretreatment and response evaluation included medical history; physical examination; complete blood counts; serum chemistry profile; computed tomography (CT) scans of the neck, chest, abdomen, and pelvis; and bone marrow biopsy and aspirate to assess the extent of disease and BCL2 rearrangement. Analysis of the molecular BCL2/IgH chimeric gene rearrangement was performed using polymerase chain reaction (PCR) according to a previously published method. ${ }^{19}$

During the follow-up physical examination, laboratory parameters and CT scans were performed every 6 months until disease recurrence or progression. Response to therapy was evaluated 6 to 8 weeks after the completion of treatment. Clinical response (including CR, unconfirmed CR, partial response $[\mathrm{PR}]$, stable disease, and progressive disease) were defined according to International Working Group recommendations. ${ }^{20}$ The frequency and severity of side effects were recorded according to the National Cancer Institute Common Toxicity Criteria (NCICTC) (version 1$).^{21}$

\section{Statistical Analysis}

A total of 54 patients entered this protocol and the intent-to-treat population included all enrolled patients. Survival from treatment (SFT) was defined as the time from the date of the first cycle of $\mathrm{FC}+\mathrm{R}$ to the date of the last follow-up examination or the date of death from any cause. Duration remission (DR) was defined as the time from the date of CR or $\mathrm{PR}$ after the completion of $\mathrm{FC}+\mathrm{R}$ cycles to the date of disease progression or the date of the last followup examination. Time to disease progression (TTP) was defined as the time from the date of the first $\mathrm{FC}+\mathrm{R}$ cycle to the date of documented disease progression or death from non-Hodgkin lymphoma or the date of the last follow-up examination. Overall survival (OS) was defined as the time from the date of first treatment after the diagnosis of FL to the date of last follow-up examination or the date of death from any cause. Survival analysis was calculated using the Kaplan-Meier method ${ }^{22}$ and statistical comparisons between the baseline characteristics of the patients and survival estimates were made using the log-rank test. ${ }^{23}$ Chi-square analysis, when appropriate, and the Fisher exact test were used to evaluate differences between baseline characteristics of the patients, in categorized form, and clinical response and toxicity related to treatment. For continuous variables, the Mann-Whitney $U$ test was used. $^{24}$ In all analyses, a 2 -sided $P$ value $<.05$ was considered to demonstrate a moderate strength of evidence against the null hypothesis. However, this level of probability was helpful to detect clinically significant variables. The Follicular Lymphoma International Prognostic Index (FLIPI) ${ }^{25}$ was calculated for 46 of the 54 patients (85\%).

\section{RESULTS Demographics}

The clinical characteristics of the 54 patients at baseline are summarized in Table 1 . The median age of the patients was 62 years (range, 44-79 years) and 59\% of the patients were female. All patients were pretreated and $20 \%$ had received $\geq 2$ prior chemotherapy regimens. Prior chemotherapy included CHOP, CHOPlike, and anthracycline plus fludarabine-containing regimens. ${ }^{26}$ Thirteen patients (24\%) had received previous treatment with fludarabine, 4 patients $(7 \%)$ had been treated previously with rituximab, and no patients had received combination treatment with chemotherapy plus rituximab. Three patients had undergone autologous stem cell transplantation. The majority of patients $(81 \%)$ had advanced disease (stage III and stage IV). The FLIPI score was $>2$ in $35 \%$ of the 
TABLE 1

Baseline Patient Characteristics

\begin{tabular}{|c|c|c|}
\hline Characteristic & No. of patients & $\%$ of total \\
\hline Total no. of patients & 54 & 100 \\
\hline \multicolumn{3}{|l|}{ Age, y } \\
\hline Median & 62 & \\
\hline Range & $44-79$ & \\
\hline \multicolumn{3}{|l|}{ Disease stage } \\
\hline $\mathrm{II}^{*}$ & 10 & 19 \\
\hline III & 18 & 33 \\
\hline IV & 26 & 48 \\
\hline \multicolumn{3}{|l|}{ Gender } \\
\hline Female & 32 & 59 \\
\hline Male & 22 & 41 \\
\hline \multicolumn{3}{|l|}{ ECOG performance status } \\
\hline 0 & 37 & 68 \\
\hline 1 & 16 & 30 \\
\hline$>1$ & 1 & 2 \\
\hline \multicolumn{3}{|l|}{ Extranodal sites of disease } \\
\hline Bone marrow & 20 & 37 \\
\hline Splenomegaly & 9 & 17 \\
\hline Liver & 3 & 6 \\
\hline \multicolumn{3}{|l|}{ FLIPI score } \\
\hline $0-1$ & 16 & 35 \\
\hline 2 & 14 & 30 \\
\hline$\geq 2$ & 16 & 35 \\
\hline Not available & 8 & \\
\hline \multicolumn{3}{|l|}{ No. of previous therapies } \\
\hline 1 & 43 & 80 \\
\hline$>2$ & 11 & 20 \\
\hline No. of patients treated with fludarabine/rituximab & 17 & 31 \\
\hline \multicolumn{3}{|l|}{ LDH IULN } \\
\hline Normal & 35 & 74 \\
\hline Elevated & 12 & 26 \\
\hline Not available & 7 & 13 \\
\hline \multicolumn{3}{|l|}{ Bulky ${ }^{\dagger}$} \\
\hline Yes & 3 & 6 \\
\hline No & 51 & 94 \\
\hline
\end{tabular}

ECOG indicates Eastern Cooperative Oncology Group; FLIPI, Follicular Lymphoma Prognostic Index; LDH, lactate dehydrogenase; IULN, institutional upper limit of normal.

* Stage II with B symptoms or bulky disease.

${ }^{\dagger}$ Lymph node mass with maximum dimension of $>5 \mathrm{~cm}$.

evaluable patients. At baseline, 45 patients were tested for BCL2 rearrangements in the bone marrow, and 29 were found to be positive.

\section{Efficacy}

Overall, 49 (92\%) of the 53 patients who began treatment completed all planned therapy in 10 to 14 weeks. Treatment was discontinued for 4 patients for the following reasons: severe and prolonged neutropenia in 3 patients (after 3 cycles in 2 patients and after 2 cycles in 1 patient) and progressive disease in 1 patient (occurring after 2 cycles). The overall response rate for all 54 patients in the intent-to-treat analysis was $90 \%$, and 40 patients (74\%) obtained a CR. Disease progression occurred in 4 patients $(7 \%)$
TABLE 2

Clinical Response by Intent-To-Treat Analysis

\begin{tabular}{lll}
\hline & No. & \% of total \\
\hline Response & & \\
Complete (CR + CRu) & 40 & 74 \\
Partial (PR) & 9 & 17 \\
Progressive disease & 4 & 7 \\
Not evaluable* & 1 & 2 \\
\hline CR indicates complete response; CRu, unconfirmed complete response; PR, partial response. \\
${ }^{*}$ One patient refused to begin therapy immediately after informed consent had been obtained. \\
\hline
\end{tabular}

and 1 patient $(2 \%)$ refused to begin therapy immediately after giving written informed consent (Table 2). A univariate analysis of baseline prognostic factors (age, sex, stage of disease, lymph node sites, hemoglobin level, lactate dehydrogenase level, FLIPI score, number of previous treatments, previous treatment with fludarabine or rituximab, BCL2-positive bone marrow involvement, and enlarged spleen) found that none of these factors were predictive of response to treatment.

At the time of last follow-up, 14 patients had died (Table 3 ). Forty patients were still alive, 31 with ongoing responses and 9 with progressive disease. The median SFT, DR, and TTP had not been reached after a median follow-up of 45 months (range, 1-74 months). Figure 2 shows the SFT, DR, and TTP curves. The associations between clinical characteristics versus SFT, DR, and TTP are summarized in Table 4 .

The probability of SFT at 4 years was $75 \%(95 \%$ confidence interval $[95 \% \mathrm{CI}], 60-85 \%)$. None of the baseline prognostic characteristics was found to be significantly correlated with SFT. The 4 -year DR rate in the 49 patients who achieved a CR or PR was $63 \%$ $(95 \%$ CI, $47-75 \%)$. Among the baseline prognostic characteristics, $<2$ previous treatments was found to be significantly $(P=.03)$ correlated with a better DR and a trend was noted in patients who were positive for BCL2 $(P=.08)$. The 4-year TTP rate in all 54 patients was $61 \%$ (95\% CI , 46-73\%). BCL2 positivity and having received $<2$ previous treatments were found to be correlated with better TTP $(P=.02$ and $P=.04$, respectively), confirming the trend observed for DR. A trend toward better TTP also was observed in patients with a FLIPI score $<2(P=.06)$. Overall survival at 8 years was $78 \%$ (95\% CI,63-88\%). Figure 2 shows the overall survival curve.

\section{PCR Assay for BCL2}

Twenty-nine of the 45 patients tested were found to be positive for BCL2 in bone marrow aspirates 
TABLE 3

Timing and Causes of Death

\begin{tabular}{lllll}
\hline Patient no. & $\begin{array}{l}\text { Time from first } \\
\text { diagnosis, mo }\end{array}$ & $\begin{array}{l}\text { Time from entry } \\
\text { into protocol, mo }\end{array}$ & Outcome & Details \\
\hline 1 & 185 & 42 & Death & Progressive disease after subsequent chemotherapy \\
2 & 34 & 29 & Death & Bladder cancer \\
3 & 34 & 18 & Death & Progressive disease after subsequent chemotherapy \\
4 & 65 & 52 & Death & Progressive disease after subsequent chemotherapy \\
5 & 86 & 29 & Death & Infection (bronchopneumonia) \\
6 & 53 & 24 & Death & Progressive disease after subsequent chemotherapy \\
7 & 104 & 39 & Death & Acute myeloid leukemia \\
8 & 33 & 3 & Death & Pancytopenia and bronchopneumonia \\
9 & 43 & 23 & Death & Progressive disease \\
10 & 49 & 48 & Death & Adrenal carcinoma \\
11 & 179 & 1 & Death & Myelodysplastic syndrome \\
12 & 13 & 37 & Death & Progressive disease \\
13 & 74 & 42 & Death & Progressive disease after subsequent chemotherapy \\
14 & 77 & Death & Progressive disease \\
\hline
\end{tabular}
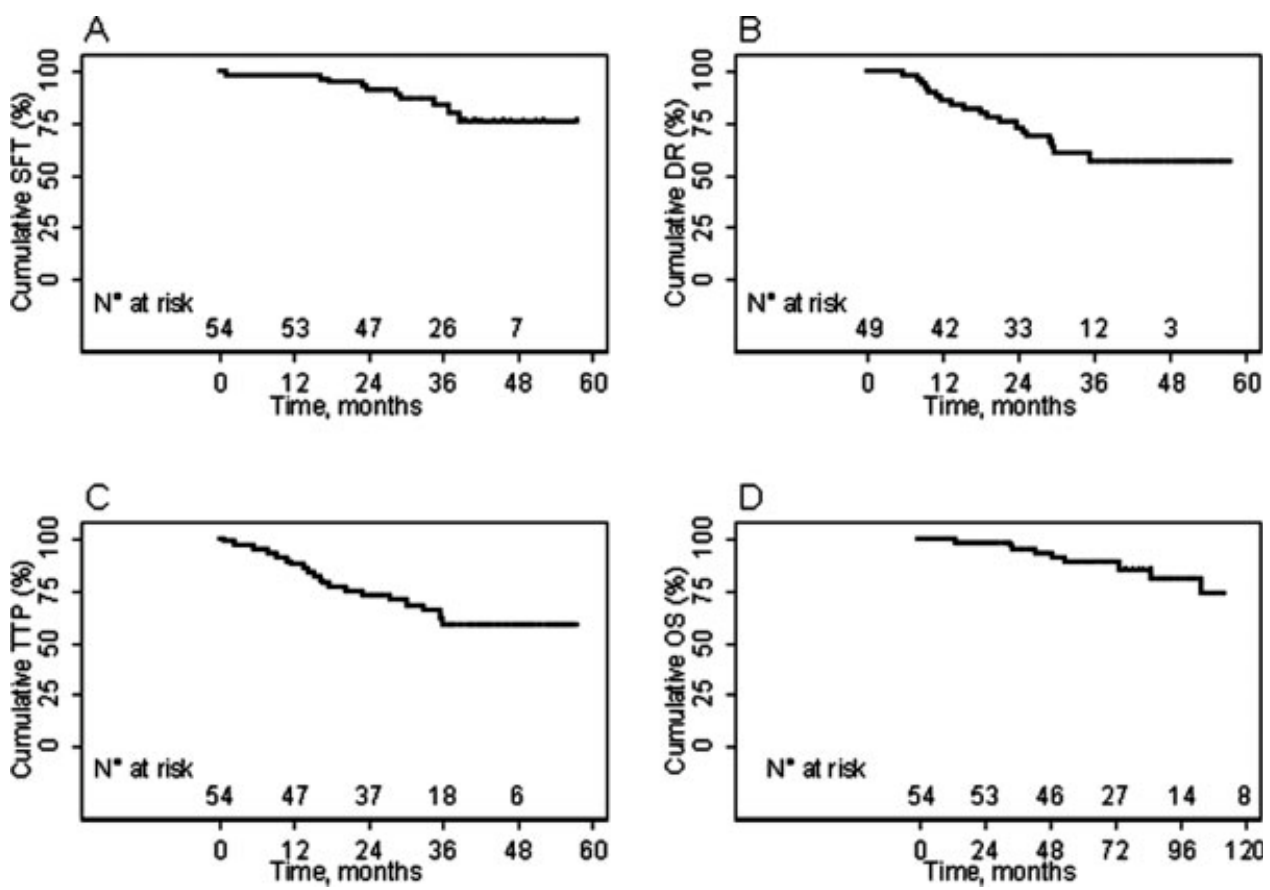

FIGURE 2. Kaplan-Meier estimates of (A) survival from treatment (SFT) (14 patients had events), (B) duration of disease remission (DR) (17 patients had events), (C) time to disease progression (TTP) (21 patients had events), and (D) overall survival (OS) (14 patients had events).

obtained prior to treatment. Of these 29 patients, 22 were evaluated at the end of treatment, 19 of whom $(86 \%)$ were converted to BCL2 negativity.

\section{Toxicity}

Toxicity was evaluable in 52 patients. Typical firstdose rituximab infusion toxicity was observed in the current study patients, as previously described. ${ }^{14}$ Fludarabine was found to be well-tolerated. The most commonly noted severe side effects were hematologic and included 21 cases of neutropenia, 3 cases of thrombocytopenia, and 2 cases of anemia (Table 5). Overall, the hematologic toxicities were transient and reversible. Treatment delays of 1 to 3 weeks were necessary in 12 patients. However, 3 patients were taken off therapy for severe and prolonged neutropenia. These 3 patients presented with WHO grade $3 / 4$ infections. Two patients recovered within 2 to 5 weeks. One patient died of bronchopneumonia 13 weeks after protocol entry. One patient was diagnosed with a myelodysplastic syndrome (refractory anemia with excess blasts) after 32 months, 
TABLE 4

Association between Clinical Characteristics Versus Survival From Treatment, Duration of Remission, and Time to Progression

\begin{tabular}{|c|c|c|c|c|c|c|}
\hline \multirow[b]{2}{*}{ Variable } & \multicolumn{2}{|c|}{ SFT } & \multicolumn{2}{|c|}{ DR } & \multicolumn{2}{|c|}{ TTP } \\
\hline & Log-rank test & $P$ & Log-tank test & $P$ & Log-rank test & $P$ \\
\hline Age, $y>60$ vs $<60$ & 2.02 & .15 & 0.19 & $>.50$ & 0.53 & .46 \\
\hline Lymph node involvement, $>4$ vs $<4$ & 0.00 & .94 & 0.79 & .37 & 1.000 & .32 \\
\hline LDH IULN, elevated vs normal & 2.50 & .11 & 0.67 & $>.50$ & 1.10 & .29 \\
\hline FLIPI, $\geq 3$ vs $\leq 2$ & 1.65 & .19 & 1.36 & .24 & 3.40 & .06 \\
\hline Stage, $\geq$ III vs $<$ III & 0.10 & .75 & 1.61 & .14 & 3.29 & .07 \\
\hline BCL2, negative vs positive & 0.12 & .72 & 2.09 & .08 & 5.12 & .02 \\
\hline Bone marrow involvement, positive vs negative & 0.30 & .58 & 0.05 & $>.50$ & 0.19 & .69 \\
\hline No. of previous therapies, $\geq 2$ vs $<2$ & 0.22 & .64 & 2.78 & .03 & 4.27 & .04 \\
\hline
\end{tabular}

SFT indicates survival from treatment; DR, duration of disease remission; TTP, time to disease progression; LDH, lactate dehydrogenase; IULN, institutional upper limit of normal; FLIPI, Follicular Lymphoma International Prognostic Index.

TABLE 5

Hematologic Toxicity* During Treatment in the 52 Evaluable Patients

\begin{tabular}{lll}
\hline & No. & \% of total \\
\hline Anemia & & \\
Grade 0 & 43 & 83 \\
Grade 1 & 6 & 11 \\
Grade 2 & 1 & 2 \\
Grade 3/4 & 2 & 4 \\
Neutropenia & & \\
Grade 0 & 22 & 42 \\
Grade 1 & 3 & 6 \\
Grade 2 & 6 & 12 \\
Grade 3/4 & 21 & 40 \\
Thrombocytopenia & & \\
Grade 0 & 41 & 79 \\
Grade 1 & 7 & 13 \\
Grade 2 & 1 & 2 \\
Grade 3/4 & 3 & 6 \\
\hline
\end{tabular}

* Toxicity was graded according to World Health Organization criteria.

and died 48 months after protocol entry (Table 3). We observed a significant association between $>4$ lymph node sites $(P=.01)$, BCL2-positive bone marrow $(P=.007)$, and female sex $(P=.01)$ with the risk of presenting with WHO grade $3 / 4$ neutropenia. Age, FLIPI score, and the number of previous treatments were not found to be associated with the grade of neutropenia observed. Only 1 patient presented with WHO grade 2 herpes zoster infection. Overall, 15 patients received G-CSF support. Prophylaxis with TMP-SMX and acyclovir was used in 10 patients and 2 patients, respectively.

\section{DISCUSSION}

The treatment of patients with FL remains a matter of controversy, and treatment choices may vary radi- cally from a watch-and-wait policy to high-dose therapy followed by autologous stem cell transplantation. However, to our knowledge, none of these options has demonstrated the potential to cure FL patients. Several treatment options are available for patients with recurrent disease, making it challenging for the treating physician to choose the best treatment. Because to our knowledge no optimal therapy has been identified to date, the characteristics of patients with recurrent disease, the type of previous treatment, the duration of previous response, age, comorbidities, and the expanding role of MoAb treatment (ie, rituximab maintenance and radiommunotherapy) must be taken into consideration during the decision-making process for the treatment of each individual patient.

A recent randomized trial compared fludarabine with the combination of cyclophosphamide, vincristine, and prednisone (CVP) in patients with recurrent FL. $^{27}$ Compared with CVP, fludarabine significantly improved progression-free survival, treatment-free survival, and social functioning scores. ${ }^{27}$ Combining fludarabine with cyclophosphamide and/or mitoxantrone could increase the efficacy of fludarabine to improve response and failure-free survival. ${ }^{28,29}$ Early phase II studies ${ }^{10}$ and recent randomized trials have demonstrated that the addition of rituximab to the CHOP regimen ${ }^{30,31}$ or to other anthracycline-containing $^{32-34}$ or nonanthracycline-containing regimens ${ }^{35}$ resulted in higher $\mathrm{CR}$ rates and better progressionfree survival rates. The rationale behind our decision to utilize the $\mathrm{FC}+\mathrm{R}$ combination was the observed in vitro synergism between fludarabine and cyclophosphamide, the ability of rituximab to sensitize lymphoma cells to fludarabine, and the ability of fludarabine to synergize with rituximab. We also considered the single-agent activity of cyclophospha- 
mide, fludarabine, and rituximab in the treatment of $\mathrm{FL}$, and the high efficacy of the $\mathrm{FC}+\mathrm{R}$ combination in the treatment of B-cell chronic lymphocytic leukemia. ${ }^{36}$

Because of the high number of older patients with comorbidities, a nonanthracycline-containing immunochemotherapy regimen would be a valid alternative treatment, especially in patients who were previously treated with CHOP. Although the use of fludarabine is associated with significant immunosuppression, ${ }^{37}$ our short-duration $\mathrm{FC}+\mathrm{R}$ scheme (only 10 weeks) could be a valuable addition to current therapeutic options because anthracycline treatment is limited by the cardiotoxicity associated with the cumulative dose. The results of the current study are encouraging and compare favorably in terms of efficacy and safety with those obtained with 6 cycles of $\mathrm{CHOP}+\mathrm{R}$ in a similar subset of patients with recurrent FL. ${ }^{31}$ Furthermore, the current study results are similar, in terms of efficacy, to those obtained in a longer-duration scheme of fludarabine plus rituximab conducted in chemotherapy-naive and recurrent FL patients. ${ }^{13}$ When evaluating the results of the current study, it is interesting to note that no patients with refractory FL were enrolled in this trial; $80 \%$ were in first disease recurrence, and $19 \%$ had stage II disease with B symptoms or bulky disease.

Approximately $89 \%$ of the patients enrolled in the current study completed all planned therapy in 10 to 14 weeks, and $74 \%$ of patients achieved a CR. At the time of last follow-up, the median SFT, DR, and TTP had not been reached after a median of 45 months, and $86 \%$ of tested patients converted to BCL2-negative bone marrow. Statistical analysis of baseline characteristics versus DR and TTP demonstrated that $<2$ previous treatments, BCL2-positive bone marrow, and low FLIPI score were correlated with better survival outcomes. Overall, hematologic toxicity was found to be reversible and a short treatment delay due to neutropenia was necessary in only 12 patients. Surprisingly, age, FLIPI score, and the number of previous treatments were not found to be associated with the grade of neutropenia. The 3 patients in whom treatment had to be discontinued because of prolonged neutropenia presented with grade $3 / 4$ infections. However, 2 of the 3 patients recovered within 2 to 5 weeks.

In conclusion, the results of the current study demonstrated that the immunotherapy scheme of fludarabine and cyclophosphamide plus rituximab had high antitumor activity, with $74 \%$ of patients achieving CRs and 89\% achieving BCL2 clearance. Treatment could be administered in $<3$ months, and the median SFT, DR, and TTP were not reached after a mean follow-up of 45 months. This treatment appears to be relatively safe, and complications from infection appeared to be a relatively rare event, despite approximately $40 \%$ of patients presenting with severe neutropenia. The current study results demonstrated that this short regimen is a possible treatment option for patients with recurrent FL and could constitute 1 treatment arm of a randomized trial. Based on the observed incidences of neutropenia, which resulted in a treatment delay in 12 patients, this scheme could be modified by cycling every 28 days instead of 21 days. Furthermore, considering the expanding role of rituximab as a maintenance therapy after induction treatment, the $\mathrm{FC}+\mathrm{R}$ regimen could be followed by rituximab infusion every 2 to 3 months for 1 to 2 years.

\section{REFERENCES}

1. The Non-Hodgkin's Lymphoma Classification Project. A clinical evaluation of the International Lymphoma Study Group classification of non-Hodgkin's lymphoma. Blood 1997;89:3909-3918.

2. Horning SJ. Natural history of and therapy for the indolent non-Hodgkin's lymphomas. Semin Oncol. 1993;9:669-687.

3. Aurora V, Winter JN. Current controversies in follicular lymphoma. Blood Rev. 2006;20:179-200.

4. Meijerink JP. $\mathrm{t}(14 ; 18)$, a journey to eternity. Leukemia. 1997;11:2175-2187.

5. Gallagher CJ, Gregory WM, Jones AE, et al. Follicular lymphoma: prognostic factors for response and survival. J Clin Oncol. 1986;4:1470-1480.

6. Adkins JC, Peters DH, Markham A. Fludarabine. An update of its pharmacology and use in the treatment of haematological malignancies. Drugs. 1997;43:1005-1037.

7. Barcos M, Bloomfield CD, Nissen NL, et al. Prolonged single-agent versus combination chemotherapy in indolent follicular lymphomas: a study of the cancer and leukemia group B. J Clin Oncol. 2003;21:5-15.

8. Rohatiner AZ, Gregory WM, Peterson B, et al. Meta-analysis to evaluate the role of the interferon in follicular lymphoma. J Clin Oncol. 2005;23:2215-2223.

9. Fisher RL, LeBlanc M, Press OW, Maloney DG, Unger JM, Miller PT. New treatment options have changed the survival of patients with follicular lymphoma. J Clin Oncol. 2005;23:8447-8452.

10. Czuczman MS, Weaver R, Alkuzweny B, Berlfein J, GrilloLopez AJ. Prolonged clinical and molecular remission in patients with low-grade or follicular non-Hodgkin's lymphoma treated with rituximab plus CHOP chemotherapy: 9-year follow-up. J Clin Oncol. 2004;22:4711-4716.

11. Liu Q, Fayad L, Cabanillas F, et al. Improvement of overall and failure-free survival in stage IV follicular lymphoma: 25 years of treatment experience at The University of Texas M.D. Anderson Cancer Center. J Clin Oncol. 2006;24:15821589 .

12. McLaughlin P, Grillo-Lopez AJ, Link BK, et al. Rituximab chimeric anti-CD20 monoclonal antibody therapy for relapsed indolent lymphoma: half of patients respond to a four-dose treatment program. J Clin Oncol. 1998;16:28252833. 
13. Czuczman MS, Koryzna A, Mohr A, et al. Rituximab in combination with fludarabine chemotherapy in low-grade or follicular lymphoma. J Clin Oncol. 2005;23:694-704.

14. Sacchi S, Federico M, Vitolo U, et al. Clinical activity and safety of combination immunotherapy with interferon- $\alpha 2 a$ and rituximab in patients with relapsed low grade non Hodgkin's lymphoma. Haematologica. 2001;86:951-958.

15. Alas S, Bonavida B. Rituximab inactivates signal transducer and activation of transcription 3 (STAT3) activity in B-nonHodgkin's lymphoma through inhibition of the interleukin 10 autocrine/paracrine loop and results in down-regulation of Bcl-2 and sensitization to cytotoxic drugs. Cancer Res. 2001;61:5137-5144.

16. Alas S, Emmanouilides C, Bonavida B. Inhibition of interleukin 10 by rituximab results in down-regulation of Bcl-2 and sensitization of B-cell non-Hodgkin's lymphoma to apoptosis. Clin Cancer Res. 2001;7:709-723.

17. Di Gaetano N, Xiao Y, Erba E, et al. Synergism between fludarabine and rituximab revealed in a follicular lymphoma cell line resistant to the cytotoxic activity of either drug alone. Br J Haematol. 2001;114:800-809.

18. Bellosillo B, Villamor N, Colomer D, Pons G, Montserrat E, Gil J. In vitro evaluation of fludarabine in combination with cyclophosphamide and/or mitoxantrone in B cell chronic lymphocytes leukemia. Blood. 1999;94:2836-2843.

19. Gribben JG, Neuberg D, Barber M, et al. Detection of residual lymphoma cells by polymerase chain reaction in peripheral blood is significantly less predictive for relapse than detection in bone marrow. Blood. 1994;83:3800-3807.

20. Cheson BD, Horning SJ, Coiffier B, et al. Report of an international workshop to standardize response criteria for non-Hodgkin's lymphomas. NCI Sponsored International Working Group. J Clin Oncol. 1999;17:1244-1253.

21. Wittes RE. Common toxicity criteria for cancer clinical trials, version 1.0. In: MacDonald JS, Haller DG, Mayer RJ, editors. Manual of Oncologic Therapeutics. 3rd ed. Philadelphia: Lippincott; 1995:445-448.

22. Kaplan EL, Meier P. Non parametric estimation from incomplete observation. J Am Stat Assoc. 1958;53:457-481.

23. Mantel N, Haenszel W. Statistical aspects of the analysis of data from retrospective studies of disease. J Natl Cancer Inst. 1959;22:719-748.

24. Mann HB, Whitney DR. On a test of whether one or two random variables is stochastically larger than the other. Ann Math Stat. 1997;18:50-60.

25. Solal-Celigny P, Roy P, Colombat P, et al. Follicular lymphoma prognostic index, Blood. 2004;104:1258-1265.

26. Luisi D, Luminari S, Amatetti C, et al. BACOP/FND firstline treatment of follicular lymphoma. Multicentric study of GISL. Preliminary result. Ann Oncol. 2004;15(suppl 12):G17.

27. Klasa RJ, Meyer RM, Shustik C, et al. Randomized phase III study of fludarabine phosphate versus cyclophosphamide, vincristine, and prednisone in patients with recurrent lowgrade non--Hodgkin's lymphoma previously treated with an alkylating agent or alkylator-containing regimen. J Clin Oncol. 2002;20:4649-4654.

28. McLaughlin P, Hagemeister FB, Romaguera JE, et al. Fludarabine, mitoxantrone and dexametasone: an effective regimen for indolent lymphoma. J Clin Oncol. 1996;14: $1262-1268$.

29. Santini G, Nati S, Spriano M, et al. Fludarabine in combination with cyclophosphamide or with cyclophosphamide plus mitoxantrone for relapsed or refractory low-grade nonHodgkin's lymphoma. Haematologica. 2001;86:282-286.

30. Hiddemann W, Kneba M, Dreyling M, et al. Frontline therapy with rituximab added to the combination of cyclophosphamide, doxorubicin, vincristine, and prednisone (CHOP) significantly improves the outcome for patients with advanced-stage follicular lymphoma compared with therapy with CHOP alone: results of a prospective randomized study of the German Low-Grade Lymphoma Study Group. Blood. 2005;106:3725-3732.

31. Van Oers MH, Klasa R, Marcus RE, et al. Rituximab maintenance improves clinical outcome of relapsed/resistant follicular non-Hodgkin's lymphoma, both in patients with and without rituximab during induction: results of a prospective randomized phase III intergroup trial. Blood. 2006;108:3295-3301. Epub 2006 Jul 27..

32. Forstpointner R, Dreyling M, Repp R, et al. The addition of rituximab to a combination of fludarabine, cyclophosphamide, mitoxantrone (FCM) significantly increases the response rate and prolongs survival as compared with FCM alone in patients with relapsed and refractory follicular and mantle cell lymphomas: results of a prospective randomized study of the German Low-Grade Lymphoma Study Group. Blood. 2004;104:3064-3071.

33. McLaughlin P, Rodriguez MA, Hagemeister FB, et al. Stage IV indolent lymphoma: a randomized study of concurrent vs. sequential use of FND (Fludarabine, Mitoxantrone, Dexamethasone) chemotherapy and rituximab monoclonal antibody therapy, with interferon maintenance. J Clin Oncol. 2003;22:564. Abstract 2269.

34. Herold M, Pasold R, Srock S, et al. Result of a prospective randomised open label phase III study comparing rituximab plus mitoxantrone, chlorambucile, prednisolone chemotherapy (R-MCP) versus MCP alone in untreated advanced indolent non-Hodgkin" lymphoma (NHL) and mantle-cell-lymphoma (MCL). Blood. 2004;104:169a. Abstract 584 .

35. Marcus R, Imrie K, Belch A, et al. CVP chemotherapy plus rituximab compared with CVP as first-line treatment for advanced follicular lymphoma. Blood. 2005;105:1417-1423.

36. Keating MJ. O'Brien S, Albitar M, et al. Early results of a chemoimmunotherapy regimen of fludarabine, cyclophosphamide, and rituximab as initial therapy for chronic lymphocytic leukemia. J Clin Oncol. 2005;23:4079-4088.

37. Wijermans PW, Gerrits WB Haak HL. Severe immunodeficiency in patients treated with fludarabine monophosphate. Eur J Haematol. 1993;50:292-296. 